\title{
Analysis of the complete genome sequence of strain Concept- 8 , the new representative of the genus Methylococcus
}

I.Y. Oshkin

Winogradsky Institute of Microbiology, Research Center of Biotechnology of the Russian Academy of Sciences ig.owkin@gmail.com

K.K. Miroshnikov

Winogradsky Institute of Microbiology, Research Center of Biotechnology of the Russian Academy of Sciences infon18@gmail.com

D.V. Chernushkin

BIOSINTEZ, LLC rs1@gkconcept.biz

N.V. Ravin

Institute of Bioengineering, Research Center of Biotechnology of the Russian Academy of Sciences, Moscow, Russia nravin@biengi.ac.ru

V.O. Popov

Research Center of Biotechnology of the Russian Academy of Sciences vpopov@inbi.ras.ru
V.N. Khmelenina

Federal Research Center "Pushchino Scientific Center for Biological

Research of the Russian Academy of

Sciences", G.K. Skryabin Institute of Biochemistry and Physiology of

Microorganisms, Russian Academy of Sciences

khmelenina@ibpm.pushchino.ru

S.E. Belova

Winogradsky Institute of Microbiology,

Research Center of Biotechnology of

the Russian Academy of Sciences svet-bel@mail.ru

A.V. Beletsky

Institute of Bioengineering, Research

Center of Biotechnology of the Russian

Academy of Sciences, Moscow, Russia mortu@yandex.ru

S.N. Dedysh

Winogradsky Institute of Microbiology, Research Center of Biotechnology of the Russian Academy of Sciences dedysh@mail.ru
S. Y. But

Federal Research Center "Pushchino Scientific Center for Biological

Research of the Russian Academy of

Sciences", G.K. Skryabin Institute of Biochemistry and Physiology of

Microorganisms, Russian Academy of Sciences

sergeybut20063@gmail.com

N.S. Khokhlachev

Gazprom VNIIGAZ gagarin88@yandex.ru

A.V. Mardanov

Institute of Bioengineering, Research Center of Biotechnology of the Russian Academy of Sciences, Moscow, Russia mardanov@biengi.ac.ru

N.V. Pimenov

Winogradsky Institute of Microbiology, Research Center of Biotechnology of the Russian Academy of Sciences npimenov@mail.ru

\begin{abstract}
The complete genome sequence of the thermotolerant obligate methanotroph and a promising singlecell protein producer Methylococcus sp. Concept-8 has been. determined and analyzed. The final genome assembly represents a circular chromosome of $3.46 \mathrm{Mbp}$. The closest phylogenetic relative of strain Concept-8 is the well-studied methanotroph Methylococcus capsulatus Bath. ANI value estimated for the genomes of these methanotrophs constituted $\mathbf{8 8 . 3 9 \%}$ which suggests that strain Concept-8 belongs to a new species of the genus Methylococcus. Both genomes contain two identical rrn operon copies, two operons pmoBAC encoding particulate methane monooxygenase and a single operon mmoXYBZDC encoding soluble methane monooxygenase. The sets of genes encoding enzymes of the $\mathrm{C} 1$ metabolism are also highly similar. In addition, we identified two prophage regions associated with prophages of the family Siphoviridae in the genome of strain Concept-8.
\end{abstract}

Keywords - methanotrophs, genome analysis, complete genome sequence, single-cell protein

\section{Motivation and aim}

\section{Motivation}

Aerobic methanotrophs are a subset of methylotrophic bacteria that can utilize methane as a sole source of energy $[1,2]$. This unique metabolic feature is widely used for production of the single-cell protein (SCP). Large-scale industrial production of SCP from natural gas using Methylococcus capsulatus BSV-874 was previously implemented in USSR. Strain Concept- 8 was obtained by long-term storage, selection, and purification of an industrial culture of Methylococcus capsulatus BSV-874. Concept-8 is considered as a promising producer of $\mathrm{SCP}$ as it demonstrates high growth rates and high biomass yield during growth on methane.

Aim

The present study aimed to determine the complete genome sequence of strain Concept-8. We focused on comparison of the genome sequence of strain Concept- 8 with that of Methylococcus capsulatus Bath in order to define precise phylogenetic position of these methanotrophs and identify shared and distinct metabolic features. In modern biotechnology, comprehensive genome analysis is necessary for defining the metabolic potential and identification of possible ways for the optimization of the metabolic capabilities

\section{Methods}

We sequenced the genome of strain Concept- 8 using both MinION nanopore (Oxford Nanopore Technologies, Oxford, UK) and Illumina MiSeq technologies (Illumina, Inc., USA). Genome annotation was performed using Prokka [3] and KEGG [4]. Genome-based phylogeny was determined based on GTDB-Tk pipeline [5].

\section{Results}

The final hybrid assembly was a circularized chromosome sequence of $3.46 \mathrm{Mbp}$, with an average $\mathrm{G}+\mathrm{C}$ content of $63.0 \%$. The closest phylogenetic relative of the strain Concept-8 is Methylococcus capsulatus Bath (98.7\% similarity of the $16 \mathrm{~S}$ rRNA gene sequences). Average nucleotide identity value estimated for these methanotrophs constituted $88.39 \%$. This indicates that strain Concept- 8 belongs to new species within the genus Methylococcus. In addition, we performed phylogenomic analysis using the 
Genome Taxonomy Database and GTDB-Tk (https://github.com/Ecogenomics/GtdbTk). It demonstrated that strain Concept-8 and Mc. capsulatus Bath clustered together within Methylococcus group. Other closest relatives were represented by Methylocaldum, Methylomagnum, Methyloterricola и Methylogaea. The compared genomes possess two copies of operons encoding rRNA and membrane methane monooxygenase, as well as one copy of operon mmoXYBZDC encoding soluble methane monooxygenase. The ability to grow on methanol is explained by the presence of two methanol dehydrogenases - MxaFJGIRACKLD and $X o x F$. The set of genes encoding the enzymes of $\mathrm{C} 1$ metabolic pathways were also highly similar. Genes encoding pyrroloquinoline quinone cofactor biosynthesis proteins, tetrahydromethanopterin-linked and tetrahydrofolatemediated pathways, and NAD-linked formate dehydrogenase were identified. The strain Concept- 8 genome encodes four isoforms of glucose-6-phosphate dehydrogenase and two 6phosphogluconate dehydrogenases/ decarboxylases that participate in oxidative ribulose monophosphate (RuMP) pathway. Mc. capsulatus Bath possesses only two isoforms of glucose-6-phosphate dehydrogenase and one 6phosphogluconate dehydrogenase. A complete set of genes involved in the function of the RuMP pathway for formaldehyde assimilation was present. Both genomes encode indicative serine pathway enzymes, serine-glyoxylate aminotransferase, hydroxypyruvate reductase and 3glycerate kinase, as well as malyl-CoA lyase and malattiokinase involved in the synthesis of glyoxylate. However, Concept-8 and Bath strains lack PEP carboxylase, one of the key enzymes of the serine pathway. Methylococcus sp. Concept-8 genome contains genes encoding the large $(c b b L)$ and small $(c b b S)$ subunits of ribulose-1,5bisphosphate carboxylase/oxygenase (Rubisco), as well as the $c b b Q$ gene encoding a polypeptide with the putative function of Rubisco's post-translational activator. Strain Concept- 8 possesses the enzymes necessary for the synthesis of glycogen: 4- $\alpha$-glucanotransferase, glucose-1- phosphate adenylyltransferase, 1,4- $\alpha$-glucan-branching enzyme, glycogen synthase and ADP-ribose pyrophosphatase. Similar cluster of genes is identified in the genome of Mc. capsulatus Bath. The genome of Methylococcus sp. Concept-8 contains 2 regions associated with prophages of the family Siphoviridae. The prophage regions found in the genome of Mc. capsulatus Bath are also homologous to those from Siphoviridae viruses. However, they differ from those found in the genome of strain Concept-8.

\section{ACKNOWLEDGMENT}

The research was supported by the Ministry of Science and High Education of Russia, grant No 075-15-2019-1830 of the Federal Research Program, unique project identifier RFMEFI60719X0297

\section{REFERENCES}

[1] Hanson R., Hanson T. (1996) Methanotrophic bacteria. Microbiol. Rev. 60: 439-471

[2] Trotsenko Y.A., Murrell J.C. (2008) Metabolic aspects of aerobic obligate methanotrophy. Adv. Appl. Microbiol. 63: 183-229.

[3] Seemann T. (2014) Prokka: Rapid prokaryotic genome annotation. Bioinformatics. 30: 2068-2069.

[4] Kanehisa M. et al. (2016) BlastKOALA and GhostKOALA: KEGG Tools for Functional Characterization of Genome and Metagenome Sequences. J. Mol. Biol. 428: 726-731.

[5] Parks, D.H. et al. (2018) A standardized bacterial taxonomy based on genome phylogeny substantially revises the tree of life. Nat. Biotechnol. 36: 996 